\title{
Focusing on the bodily self: The influence of endogenous attention on visual body processing
}

\author{
Clara Aranda, María Ruz, Pío Tudela, and Daniel Sanabria \\ University of Granada, Granada, Spain
}

\begin{abstract}
The present study explores whether endogenous attention can modulate body perception. A modified version of the Posner paradigm was used to direct participants' attention toward the appearance of distinct body images, which differed only in detailed idiosyncratic features: one's own and another person's hands. Hand stimuli were preceded by symbolic cues that predicted their identity with high probability, which made it possible to compare the processing of expected (valid) and unexpected (invalid) targets. Results revealed that endogenous attention influenced the processing of participants' own hands by speeding participants' responses to valid in contrast to invalid trials. Crucially, no validity effect was found for the hands of another person. These findings cannot be explained in terms of perceptual familiarity, since an optimization of the processing for both familiar and unfamiliar faces by symbolic cues was observed. In light of these results, it is suggested that participants are able to anticipate particular stimuli within the same perceptual category as long as these stimuli appear to be remarkably distinct to them, which is probably the case for particular faces and their own bodies, in contrast to other people's bodies.
\end{abstract}

Over recent decades, the notion of self has become a central issue in experimental psychology and cognitive neuroscience (Gillihan \& Farah, 2005; Northoff et al., 2006). Despite its complexity and multiple dimensions (Damasio, 1999, 2003; Gallagher, 2000; James, 1890; Neisser, 1988), it is undisputed that the self constitutes a unique phenomenological experience. But can we capture this subjective uniqueness with objective measures? Experimental evidence provides a positive answer to this question, since self-related information seems to be processed in a distinct and special way, in contrast to nonself-related information (e.g., Kircher et al., 2000; see Gillihan \& Farah, 2005, for a review).

The experience of a normal self includes the feeling of residing within the limits of one's body (Blanke \& Arzy, 2005). In fact, the perception of one's body is considered a seminal stage in the emergence of the self (Damasio, 1999; Gallagher, 2000; James, 1890), since the perception of intero- and exteroceptive stimuli and their linkage to a particular subjectivity is thought to be at the basis of one's own awareness. This bodily self seems to sustain a special status for visual perception (cf. Daprati, Wriessnegger, \& Lacquaniti, 2007b; Frassinetti, Maini, Romualdi, Galante, \& Avanzi, 2008; Knoblich \& Flach, 2003).

The visual processing of our own body comprises two dissociable aspects - motion and physical appearance (see, e.g., Frassinetti et al., 2009) — that have been approached by different areas of research. In the literature on body motion perception, several studies have shown that self-generated movements are processed differently from someone else's movements and that the former are closely related to motor cognition (Calvo-Merino, Grèzes, Glaser, Passingham, \& Haggard, 2006; Daprati \& Sirigu, 2002; Knoblich \& Flach, 2003). This different processing typically leads to a better discrimination of the kinematic parameters of our own movements in comparison with others' movements, which takes place within awareness (explicit self-recognition: Daprati, Wriessnegger, \& Lacquaniti, 2007a; Jokisch, Daum, \& Troje, 2006; Loula, Prasad, Harber, \& Shiffrar, 2005) and outside it (enhanced perceptual performance: Daprati et al., 2007b; Knoblich \& Flach, 2003).

Research on static images or self-appearance has mainly focused on face recognition. Behavioral studies have shown that the recognition of one's own face is consistently faster than the recognition of a stranger's face, both in typical (upright, front, three-quarters) or atypical (upside-down, profile) views, even after hundreds of presentations (Tong \& Nakayama, 1999). However, the main goal of this line of research has been to determine the different neural underpinnings of perceiving our own face in comparison with other faces, which has highlighted a specific neural network for self-face stimuli (Kircher et al., 2000; Sugiura et al., 2000; Sugiura et al., 2006; Sugiura et al., 2005). The visual recognition of headless bodies or other different body parts has evoked considerably less interest in researchers. However, the few existing studies have revealed some interesting findings that resemble the previously mentioned results in body motion perception (see Daprati et al., 2007b). For instance, Frassinetti et al. (2008) identified a self-advantage effect that led to a faster and more ac-

C.Aranda, claraac@ugr.es 
curate match-to-sample performance for participants' own versus extraneous body images (hands and feet: Frassinetti et al., 2010; Frassinetti et al., 2008). In addition, recent fMRI studies have revealed the neural specificity of selfbody processing (for headless bodies, see Hodzic, Kaas, Muckli, Stirn, \& Singer, 2009; Hodzic, Muckli, Singer, \& Stirn, 2009; for hands, see Myers \& Sowden, 2008).

Taken together, the findings described above suggest that our own body receives special processing, which fulfills the criteria of functional independence and anatomical specificity proposed by Gillihan and Farah (2005). Nevertheless, it is important to note that the above-mentioned studies have emphasized the distinctiveness of body visual perception by means of stimulus-driven effects. That is, all these studies have identified a self-specific pattern of data that emerged from the direct presentation of one's own and someone else's body displays (e.g., Frassinetti et al., 2008; Hodzic, Kaas, et al., 2009; Hodzic, Muckli, et al., 2009; Myers \& Sowden, 2008). Here, a novel approach is proposed to assess this issue: How do expectations about one's own or someone else's body influence their subsequent processing?

Endogenous attention is understood as the ability to voluntarily focus cognitive resources on a specific attribute of a stimulus. This form of attention has mainly been studied using symbolic cues, whose interpretation enables participants to intentionally direct their attention to the cued attribute (e.g., Posner, 1980). The preparatory state triggered by the symbolic cue can be referred to various dimensions of the upcoming stimulus, such as its spatial location, temporal occurrence, color, direction of motion, or even the semantic meaning of linguistic stimuli. This preparatory state or expectation is known to optimize the subsequent processing of the target (e.g., Correa, Lupiáñez, Madrid, \& Tudela, 2006; Griffin, Miniussi, \& Nobre, 2001; Krauzlis \& Adler, 2001; Ruz \& Nobre, 2008; Vierck \& Miller, 2008; Vossel, Weidner, Thiel, \& Fink, 2009), probably by providing a competitive bias that favors task-relevant representations over competing representations (Bar, 2003; Stokes, Thompson, Nobre, \& Duncan, 2009).

The present study explores whether symbolic cues that prepare participants to process a visual image of their own hands or someone else's hands can enhance the processing of expected (valid) as compared with unexpected (invalid) hand images. Thus, the rationale of the study differs from previous research on body perception, since it explores the effects on visual body processing of orienting attention to different body images, which goes beyond mere visual presentation.

The present study also represents interesting progress in research on attention, since the effect of preparatory states regarding the perceptual appearance of specific objects remained unexplored until recently (see Lauwereyns, 1998). Few studies have assessed whether expectations about different categories (e.g., faces, houses, or words) can optimize the processing of upcoming stimuli belonging to these categories (Aranda, Tudela, Madrid, \& Ruz, 2010; Esterman \& Yantis, 2010; Puri, Wojciulik, \& Ranganath, 2009). However, the authors of the present study are aware of only two studies that have explored whether endogenous attention can be devoted to particular stimuli within a perceptual category (Faulkner, Rhodes, Palermo, Pellicano, \& Ferguson, 2002; Puri \& Wojciulik, 2008). These studies showed that cues that anticipate the specific identity of stimuli are able to optimize the visual processing of their corresponding images. The present study extends these findings, given that the two expected items that belong to the same perceptual category differ exclusively in very fine-grained features.

Therefore, the present study had a double objective. One objective was to assess the special quality of the bodily self from an innovative perspective, testing whether one's own/others' expectations exert a different influence on visual body processing (images of hands). The second objective was to explore whether endogenous attention can be focused on idiosyncratic stimuli that belong to a single perceptual category.

A modified version of the Posner attentional paradigm with symbolic predictive cues was used to induce expectations about the subsequent appearance of either one's own or someone else's hands. Cues were predicted to enhance the processing of stimuli (one's own and another person's hands) in valid compared with invalid trials. In addition, given that previous research has shown that our own body holds a distinctive status (e.g., Frassinetti et al., 2008), it was hypothesized that attention might have a greater effect on participants' own hands than on others' hands.

\section{EXPERIMENT 1}

The aim of Experiment 1 was to study whether expectations about one's own and others' hands can modulate their subsequent visual processing.

\section{Method}

Participants. Twenty-four participants (14 female; mean age $=$ 26 years; range $=21-32$ years) from the University of Granada took part in Experiment 1. All of them had normal or corrected-tonormal vision, provided informed consent prior to participation in the experiment, and received course credits in exchange for their participation.

Apparatus and Stimuli. Stimuli consisted of a fixation point $(+)$, a colored cue (a red or blue star) sustaining $3.8^{\circ}$ of angular size, and a target hand, framed in an area that measured $450 \times$ 601 pixels.

Target stimuli consisted of color images of left and right hands presented in an upright position (egocentric perspective) on a black background. The hands of each participant were photographed with a digital camera in a session prior to the experiment. This session took place in a controlled environment with constant artificial light and a fixed distance between the camera lens and the hands $(40 \mathrm{~cm})$, which were always photographed in the same horizontal position. Subsequently, photographs were modified with Adobe Photoshop software: They were cut from the original picture, pasted on a black background, and reoriented into a vertical position. Other people's hands were selected from this database as the best match for size, skin color, age, and gender, in comparison with each participant's hands. The sizes of the hands were compared in the pictures. The mean average of the differences between matched hands was $\pm 89 \mathrm{~mm}$ in length and $\pm 58 \mathrm{~mm}$ in width. In addition, the ages of the people whose hands were matched with the participants' hands 
varied within 0 to 4 years of the participants' ages. This criterion was adopted to prevent participants from using holistic features to orient attention, and to favor expectations based on the stimulus idiosyncrasy instead. In each session, target stimuli consisted of two pairs of hands (left and right) obtained from the corresponding participant and an unidentified single individual (one's own and another person's hands).

All stimuli were presented centrally on a 17-in. Dell screen controlled by E-Prime Version 2.0 software (Psychology Software Tools, Pittsburgh, PA). The PC screen was approximately $50 \mathrm{~cm}$ from the participants.

Design and Procedure. The experiment used a withinparticipants design with the factors target identity (one's own vs. another person's hands) and validity (valid vs. invalid trials).

Every trial involved presenting a fixation point of a random duration between 300 and $700 \mathrm{msec}$, followed by a colored star for $200 \mathrm{msec}$. After an interstimulus interval of $1,300 \mathrm{msec}$, the target appeared for 1,200 msec. Figure 1 shows the temporal course of a single trial. The colored cue predicted the nature of the target on $80 \%$ of the trials (valid trials). For half of the participants, the red cue predicted the appearance of their own hand and the blue cue predicted the appearance of the other person's hand. The reverse was true for the remaining 12 participants. Once the target hand was shown, participants had to discriminate whether it was a left or right hand. Participants responded to left-hand stimuli by pressing the " $\mathrm{z}$ " key on the keyboard with their left index finger and to right-hand stimuli by pressing the " $\mathrm{m}$ " key with their right index finger. This specific stimulus-response correspondence was maintained for all participants, to prevent Simon interference effects. Participants were instructed to respond as quickly as possible while trying to avoid errors. The experiment consisted of a practice block of 40 trials followed by six experimental blocks of 40 trials each ( 240 experimental trials, total). Within each block of trials, half of the stimuli were participants' own hands and the other half were others' hands. Participants were informed in advance about color-target hand associations (cue validity), but not about the presence of invalid trials in the design. They were explicitly asked to maintain their gaze on the center of the screen and to avoid looking at their hands during the entire experimental session. Short breaks were allowed between blocks.

Prior to the beginning of the experiment, participants performed a recognition block of 80 trials (50\% their own and 50\% someone else's stimuli). Its purpose was to expose participants to their own and someone else's hands, to ensure that they were able to discrimi- nate between them and to facilitate the subsequent attentional orienting to specific hand stimuli in the testing session. Participants were asked to press a key only in response to own-hand stimuli (a go/ no-go task). Those who did not reach $80 \%$ accuracy in this block ( 5 out of 72 participants across the three experiments reported in this article; $5.5 \%$ of the entire sample) were exempted from participating. The whole experimental session lasted about $30 \mathrm{~min}$.

\section{Results and Discussion}

Reaction times (RTs) shorter than $100 \mathrm{msec}$ and longer than 1,200 $\mathrm{msec}$, data from practice trials, and errors were excluded from the analysis (see Table 1).

A repeated measures ANOVA was conducted on the participants' mean RTs. The ANOVA revealed only a significant interaction between target identity and validity $\left[F(1,23)=7.46, M S_{\mathrm{e}}=436, p=.012\right]$. Subsequent a priori comparisons revealed that participants responded more quickly to valid than to invalid trials exclusively for their own hands (521 vs. $539 \mathrm{msec} ; M S_{\mathrm{e}}=423, p=.004$ ), whereas the difference in RT between valid and invalid trials for others' hands was not statistically significant [531 vs. $527 \mathrm{msec}(F<1)$; see Figure 2]. The same two-factor ANOVA on participants' error ratings did not reveal any significant effects.

The results of Experiment 1 showed that endogenous attention can influence the visual processing of body images (hands). Crucially, endogenous orienting did not improve the processing of every expected stimulus. Attention influenced the processing of participants' own hands, whereas no validity effect was found for the hands of others. In any case, this finding suggests that attention, at least under certain circumstances, can be oriented to particular items on the basis of detailed idiosyncratic features. However, it should be noted that this differential effect for stimuli from oneself as opposed to stimuli from another person admits several interpretations.

The results of Experiment 1 could be explained by an essential distinctiveness between one's own and someone

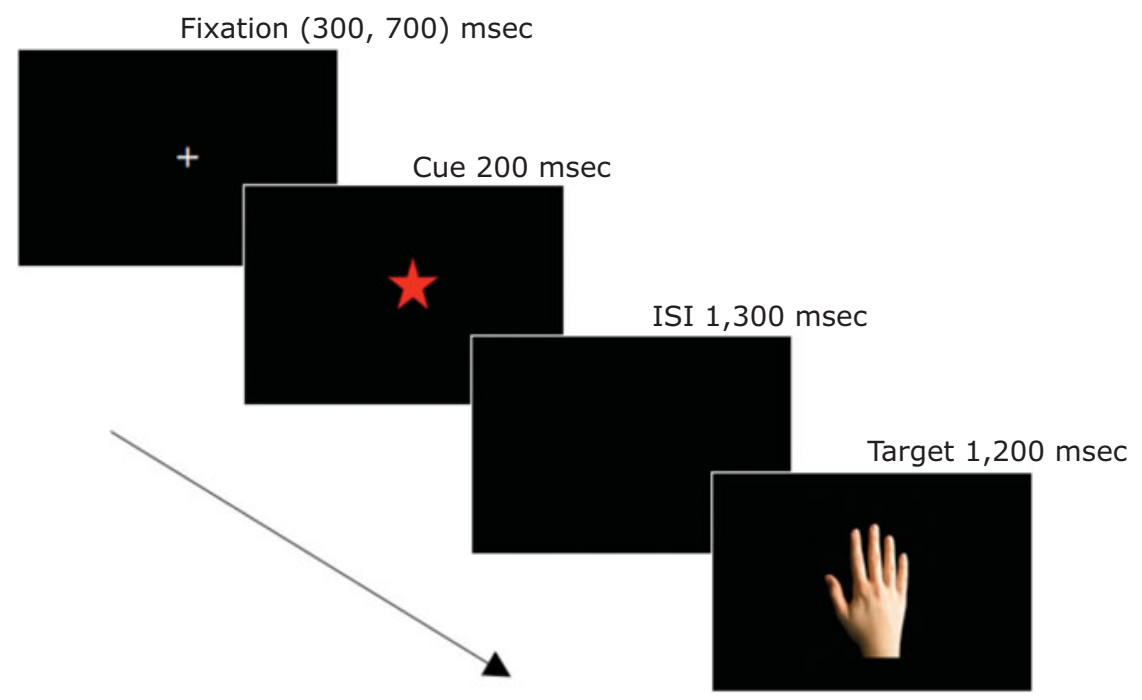

Figure 1. The sequence of events in a trial. ISI, interstimulus interval. 
Table 1

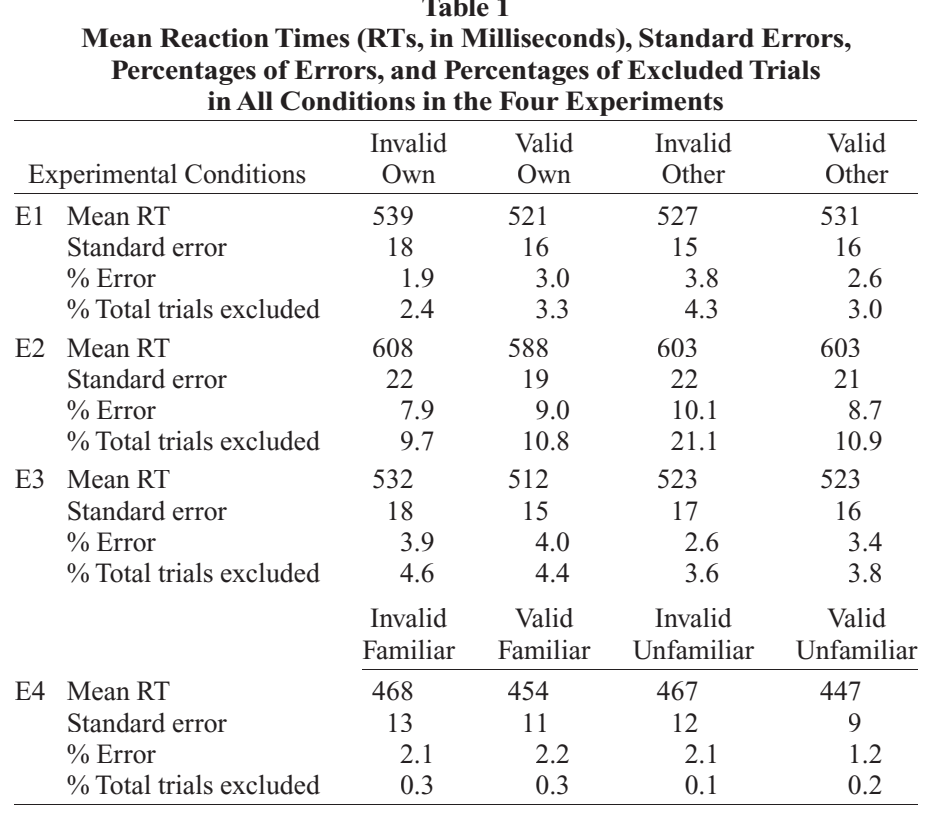

else's hands. It seems plausible that stimuli that represent one's own hand sustain a more robust internal representation in contrast to stimuli that are someone else's hand (see Tong \& Nakayama, 1999), which would have induced a greater attentional effect on target processing. Thus, the present results might reflect the existence of critical differences between one's own and others' hands, which influence the capacity of endogenous attention to optimize their subsequent visual processing.

Alternatively, these results could be explained in terms of pure manual response facilitation, rather than an optimization of processing triggered by endogenous attention. The manual response facilitation could have emerged as a result of the direct match between expectation (owncolored star), stimulus (one's own hand), and response (performed by the same "own" hand just perceived) present in valid "own" trials. This match could have led to a significant difference between valid and invalid trials exclusively for own-hand stimuli, since the same full stimulus-response congruency is not present for hand stimuli of others. Before drawing any firm conclusion, we performed a second experiment to test this alternative account.

\section{EXPERIMENT 2}

In Experiment 2, the manual response was replaced by a vocal response to test whether the results of Experiment 1 were due to the influence of endogenous attention on target processing or, alternatively, to response facilitation processes.

\section{Method}

Participants. Twenty-four participants (12 female; mean age $=25$ years; range $=18-32$ years) from the University of Granada took part in Experiment 2. All of them had normal or corrected-to- normal vision, provided informed consent prior to participation, and received course credits in exchange for their participation.

Apparatus, Stimuli, Design, and Procedure. The apparatus, stimuli, design, and procedure were the same as in Experiment 1 except for the following: Participants were required to give a vocal response for left-right judgments using a microphone connected to the PC. They kept their hands on their knees, under the black desk that supported the PC screen and the microphone. During the experimental session, the examiner stayed inside the room but out of sight of participants, to code the accuracy of responses.

\section{Results and Discussion}

The same exclusion parameters were applied as in Experiment 1: Only RTs between 100 and 1,200 msec and correct responses from the six experimental blocks were

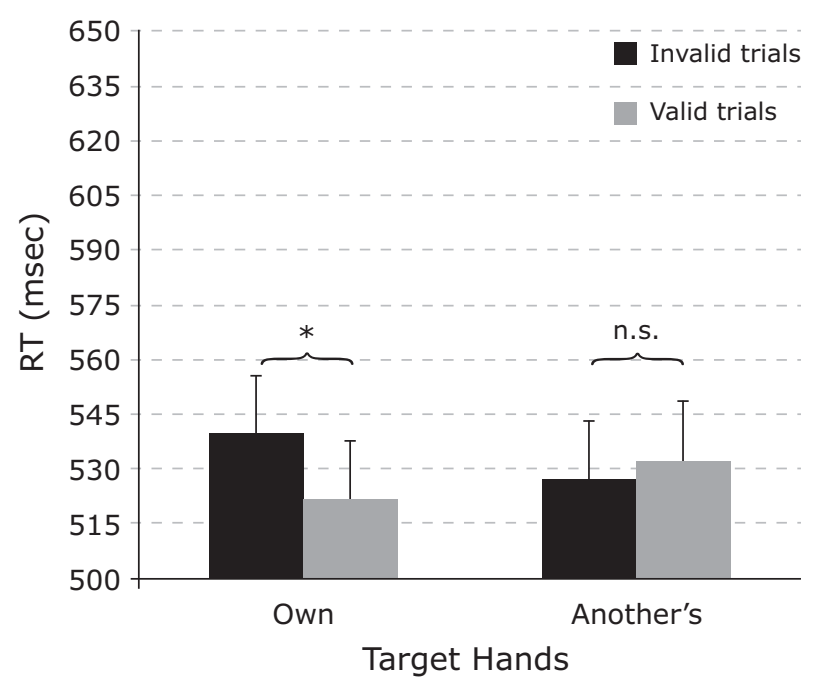

Figure 2. Validity effects on mean RTs for one's own and another's hands in Experiment 1 (manual response). 


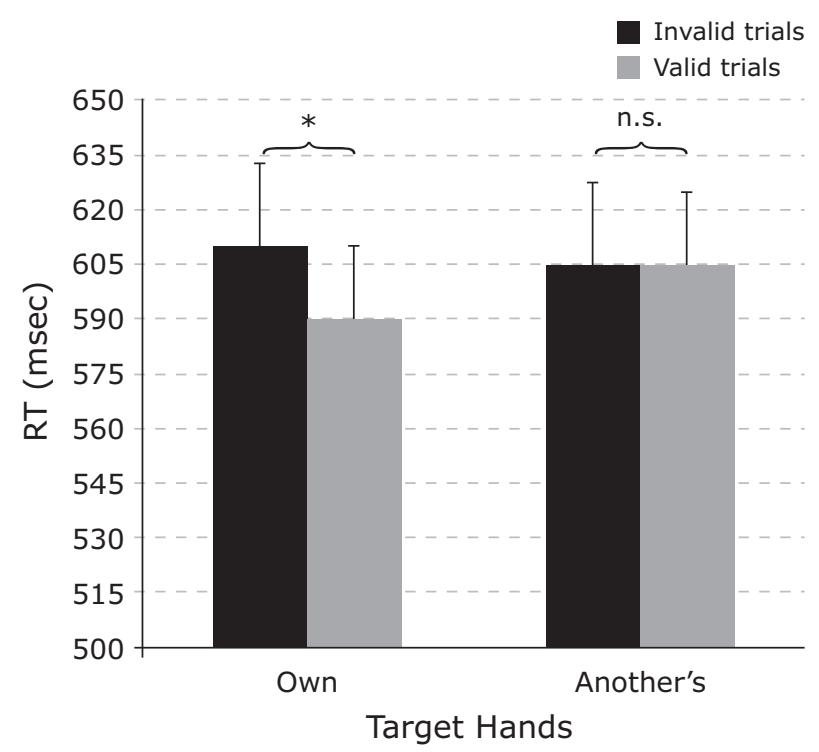

Figure 3. Validity effects on mean RTs for one's own and another's hands in Experiment 2 (vocal response).

considered for statistical analysis (see Table 1 for the percentage of trials excluded).

The ANOVA on RT data revealed a significant interaction only between target identity and validity $[F(1,23)=$ $\left.6.53, M S_{\mathrm{e}}=373, p=.019\right]$ (see Figure 3). Further analyses showed a significant difference between valid and invalid trials for own-hand targets ( 588 vs. $608 \mathrm{msec}$, respectively; $M S_{\mathrm{e}}=554, p=.007$ ), whereas no effect was obtained for targets involving others' hands $[603 \mathrm{msec}$ for valid trials vs. $603 \mathrm{msec}$ for invalid trials $(F<1)]$. The analysis of error data did not reveal statistically significant results.

The results of Experiment 2 suggest that the response facilitation hypothesis does not account for the results obtained in Experiment 1. Thus, these results support the hypothesis of a differential modulation of the processing of one's own hands with respect to others' hands as a result of endogenous orienting. However, there is an aspect in the design of Experiments 1 and 2 that might be crucial to explaining the present results. In both experiments, participants performed a recognition block prior to the experimental procedure in which they had to respond only to their own hands. Therefore, an arbitrary bias to self-stimuli may have been established as a result of these explicit instructions. To test this new alternative hypothesis, we had participants respond exclusively to hands of others in the recognition block of Experiment 3. If the dissociation found in Experiments 1 and 2 is caused by an arbitrary bias to self-stimuli, Experiment 3 should show an attentional modulation restricted to stimuli involving others. However, if the effect is due to an essential distinctiveness of participants' own hands, the results reported previously should be replicated.

\section{EXPERIMENT 3}

Experiment 3 tested a different explanation of the selfspecific attentional effect reported in Experiments 1 and 2.
To rule out the presence of an induced bias to self-stimuli, the recognition go/no-go task was modified to require participants to respond only to someone else's hands.

\section{Method}

Participants. Twenty-four participants ( 20 female; mean age $=$ 21 years; range $=19-29$ years) from the University of Granada took part in Experiment 3. All of them had normal or corrected-tonormal vision, provided informed consent prior to participation, and received course credits in exchange for their participation.

Apparatus, Stimuli, Design, and Procedure. These were the same as in Experiment 1 except for the following: In the recognition block, participants were required to discriminate between the stimuli by responding exclusively to the hands of others.

\section{Results and Discussion}

The criteria used for data exclusion were the same as in Experiments 1 and 2: Only RTs between 100 and $1,200 \mathrm{msec}$ and correct responses from the six experimental blocks were included in the statistical analysis (see Table 1 for the percentage of trials excluded).

The ANOVA of the participants' mean RT revealed a significant main effect of validity $\left[F(1,23)=8.33, M S_{\mathrm{e}}=\right.$ $293, p<.01]$. Crucially, this validity effect was modulated by target identity, as revealed by the significant interaction between the two factors $\left[F(1,23)=4.47, M S_{\mathrm{e}}=463, p<\right.$ .05] (see Figure 4). Subsequent a priori comparisons replicated the findings of Experiments 1 and 2: Endogenous orienting to self-stimuli speeded participants' responses in valid trials as compared with invalid trials for their own hands (512 vs. $\left.532 \mathrm{msec} ; M S_{\mathrm{e}}=312, p<.001\right)$. In contrast, no significant RT differences were obtained between valid and invalid trials for others' hands [523 $\mathrm{msec}$ for valid trials vs. $523 \mathrm{msec}$ for invalid trials $(F<1)]$. Error ratings did not show any significant difference across variables.

A further between-experiments ANOVA was carried out to compare participants' performance among the three experiments. The factor target type (right vs. left hand)

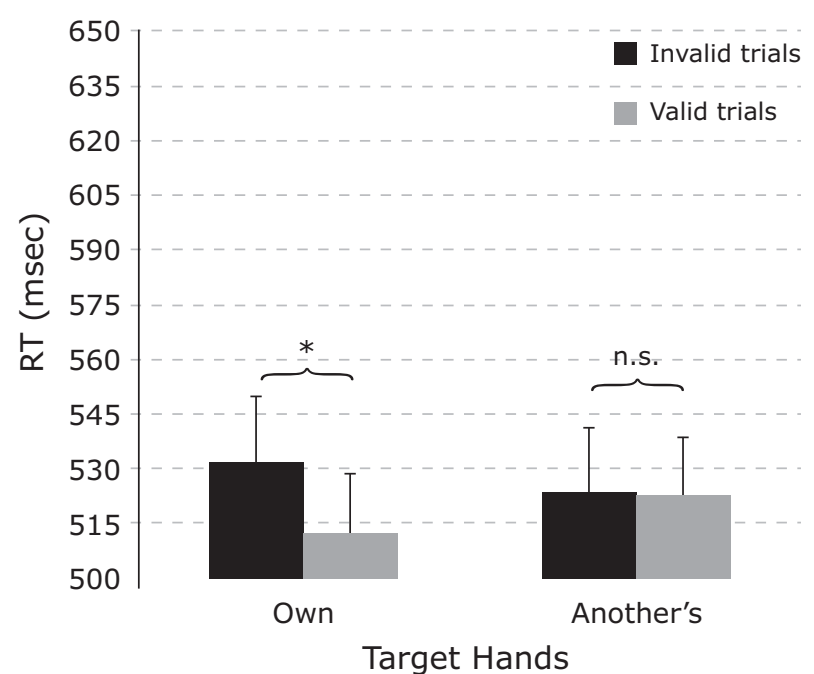

Figure 4. Validity effects on mean RTs for one's own and another's hands in Experiment 3 (reversed recognition block). 
was also included, to explore whether participants were expecting a specific hand image (right or left) within identities. Thus, the analysis was performed with the factors experiment, validity, target identity, and target type. This ANOVA revealed a significant main effect of experiment $\left[F(2,69)=5.62, M S_{\mathrm{e}}=31,867, p<.01\right]$, showing a longer mean RT in Experiment 2 (vocal response) than in Experiments 1 and 3 (manual response). These RT differences between manual and vocal responses have been shown repeatedly in previous research (e.g., Baron \& Journey, 1989; Doose \& Feyereisen, 2001; Nebes, 1978; Wells, 1924) and response modality does not seem to alter the experimental findings (e.g., Borkenau, Paelecke, \& $\mathrm{Yu}, 2010)$. The main effect of validity and the interaction between validity and target identity also reached statistical significance $\left(M S_{\mathrm{e}}=573, p=.002\right.$, and $M S_{\mathrm{e}}=424, p<$ .001 , respectively). Once again, subsequent comparisons revealed that validity effects were confined to processing of participants' own hands $\left[F(1,69)=31, M S_{\mathrm{e}}=429\right.$, $p<.001$; for others' hands, $F<1]$. Crucially, the significant interaction between target identity and validity was not modulated by the experiment factor $(F<1)$. In addition, none of the interactions involving validity and target type reached statistical significance [validity $\times$ target type, $F(1,69)=0.01, p=.91$; target identity $\times$ validity $\times$ target type, $F(1,69)=0.001, p=.97$; experiment $\times$ target identity $\times$ validity $\times$ target type, $F(2,69)=0.2, p=.75]$, suggesting that expectations were not biased toward a left or right image of the hands.

The results of Experiment 3 seem to confirm that the self-specific attentional effect is independent of arbitrary biases caused by the previous go/no-go task. The selfadvantage may thus be based on the particular distinctiveness of own-hand stimuli. Nevertheless, it is necessary to consider a further alternative explanation related to the two types of stimuli used in the present set of experiments. Our own hands are highly familiar in comparison with the hands of a stranger. Therefore, the identified effect may be related to the perceptual familiarity of stimuli rather than a special distinctiveness of self-related stimuli. To assess this issue, we performed a further experiment to test the influ-

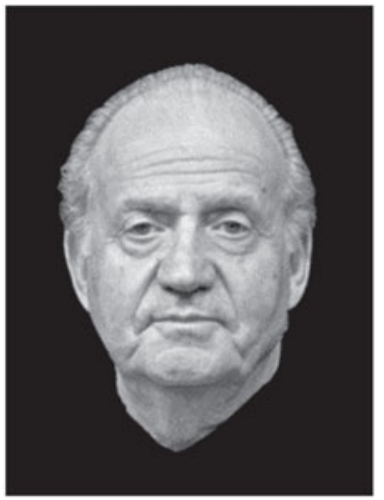

Familiar Face ence of perceptual familiarity or prior experience with the target stimulus in the generation of idiosyncratic expectancies and their subsequent effect on visual processing.

\section{EXPERIMENT 4}

Experiment 4 tested whether the identified self-specific attentional effect reported in Experiments 1-3 may be due to the high perceptual familiarity of one's own hands as stimuli, in comparison with someone else's hands. To clarify self-reference and familiarity factors, a highly familiar face that was not personally related to the participants was chosen (the king of Spain), as well as a matched unknown face, which was perceptually similar in terms of hair color, age, front view, direct gaze, and neutral emotional expression (see Figure 5). Face stimuli were selected because they belong to a body category (like hand stimuli) and they are highly discriminable in terms of perceptual familiarity. In addition, previous research has shown that participants can create expectations about faces, both as a general category and as particular stimuli belonging to famous people (e.g., Aranda et al., 2010; Faulkner et al., 2002; Puri \& Wojciulik, 2008).

\section{Method}

Participants. Twenty-four participants ( 15 female; mean age $=$ 21 years; range $=17-24$ years) from the University of Granada took part in Experiment 4. All of them had normal or corrected-tonormal vision, provided informed consent prior to participation, and received course credits in exchange for their participation.

Apparatus, Stimuli, Design, and Procedure. These were the same as in Experiment 1 except for the stimuli used and the task that had to be performed on the targets. Two colored faces were used (see Figure 5 for a black-and-white display of the faces), with one of their ears removed (left or right). Participants were asked to respond to the side in which the ear was missing by pressing " $\mathrm{z}$ " for left absence and " $\mathrm{m}$ " for right absence. This task was selected because of its similarity with the left-right decision performed on the hands in the previous experiments. Participants completed a Likert scale of familiarity ratings $(1=$ not familiar at all; $5=$ highly familiar $)$ for both faces prior to the beginning of the experiment (average ratings for the familiar face $=5$; average ratings for the unfamiliar face $=$ 1.6). All participants explicitly recognized the familiar face's identity as the king of Spain.

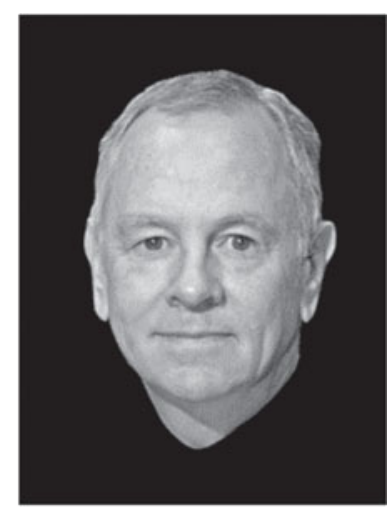

Unfamiliar Face

Figure 5. Familiar and unfamiliar face displays. 


\section{Results and Discussion}

The criteria used for data exclusion were the same as in Experiments 1 to 3: Only RTs between 100 and 1,200 msec and correct responses from the six experimental blocks were included in the statistical analysis (see Table 1 for the percentage of trials excluded). Data from 1 of the participants were also excluded due to a computer error recording her responses.

The ANOVA revealed only a significant main effect of validity $\left[F(1,22)=10.35, M S_{\mathrm{e}}=647, p<.01\right]$. Crucially, no interaction was observed between validity and target identity factors $\left[F(1,22)=1.52, M S_{\mathrm{e}}=175, p=.2\right]$, which stands in sharp contrast to the previously reported experiments. Subsequent a priori comparisons showed that attentional expectations influenced both familiar and unfamiliar faces, which was reflected in faster RTs to valid than to invalid trials [familiar face, 454 vs. $468 \mathrm{msec}$ $(p<.05)$; unfamiliar face, 447 vs. $467 \mathrm{msec}(p<.01)]$. (See Figure 6.)

Results of Experiment 4 showed that participants were able to generate endogenous expectations about particular well-known faces (as previously shown by Faulkner et al., 2002; Puri \& Wojciulik, 2008) and unknown faces. Responses were faster for valid than for invalid trials, and, importantly, this effect did not depend on the degree of familiarity of the faces. This result indicates that mere differences in the perceptual familiarity of the targets cannot account for the divergence between the validity effects for one's own and someone else's hands reported in Experiments 1-3.

\section{GENERAL DISCUSSION}

The set of experiments reported in this article explored for the first time whether symbolic cues that predict the appearance of our own hand or another person's hand can optimize the processing of these stimuli. Results showed that participants could prepare in advance to see their own hands, since cue validity influenced their performance by speeding up RTs with expected in comparison with unexpected own-hand stimuli. Interestingly, no attentional effect was observed for the hands of other people, which suggests that participants could not use the cue to generate an expectation for those specific stimuli. Experiments 2 and 3 tested alternative hypotheses to explain these findings, which ruled out explanations in terms of manual response facilitation and an arbitrary bias to own-hand stimuli, respectively. Crucially, the results of Experiments 2 and 3 replicated those of Experiment 1. Finally, Experiment 4 tested whether this selective attentional effect could be due to the higher perceptual familiarity of one's own hands with respect to other people's hands by replacing the stimuli with a highly familiar face (the king of Spain) and a nonfamiliar face. Results showed that participants could use the cues to anticipate the appearance of both faces, since a behavioral advantage was observed for all valid face stimuli regardless of their degree of familiarity. Therefore, the results of Experiments 1-3 cannot be explained in terms of mere perceptual familiarity.

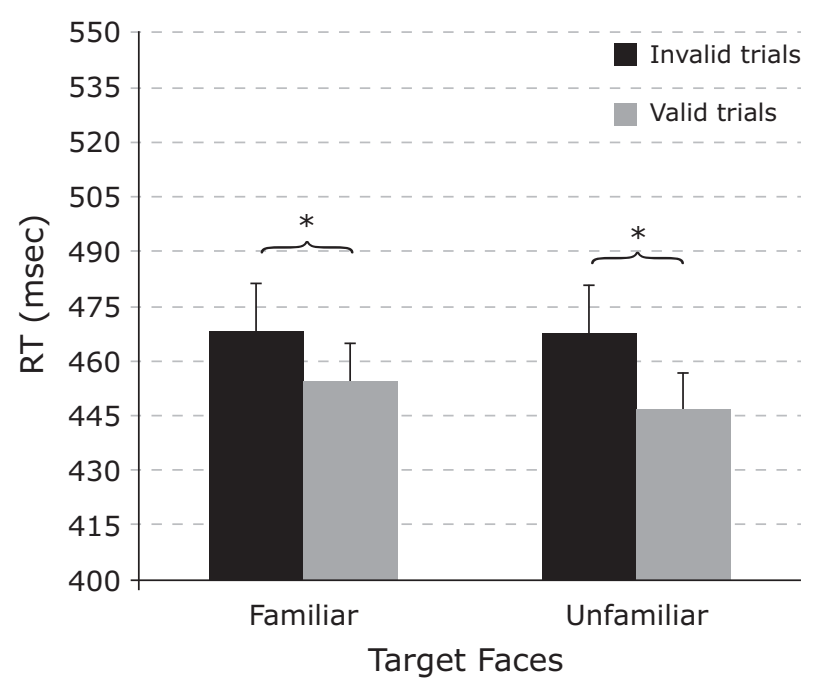

Figure 6. Validity effects on mean RTs for familiar and unfamiliar faces in Experiment 4 (perceptual familiarity).

A key question arising from such results is why no attentional effect was observed with someone else's hands. Previous research has shown that devoting attention to general stimulus attributes can optimize target processing regardless of stimulus type (Aranda et al., 2010; Esterman \& Yantis, 2010; Griffin et al., 2001; Krauzlis \& Adler, 2001; Puri et al., 2009; Ruz \& Nobre, 2008; Vierck \& Miller, 2008; Vossel et al., 2009). This could have led one to expect validity effects for nonself stimuli in the present paradigm. However, the vast majority of previous studies did not require participants to expect particular items within a single category (e.g., participants were required to attend to a spatial location). This crucial difference between the present experiments and previous studies may explain the divergence of findings.

Regarding expectations about particular stimuli, recent studies have revealed that attention influences task performance on expected items when using well-known stimuli (famous faces and buildings; Faulkner et al., 2002; Puri \& Wojciulik, 2008). Given that Experiment 4 showed that nonfamiliar face stimuli also benefit from this specific orienting, it is considered plausible to explain these findings in terms of stimulus distinctiveness. Idiosyncratic expectations are based on very subtle perceptual differences that need to be available to the participants prior to the presentation of stimuli. It is suggested that participants are able to create idiosyncratic expectations only when these relate to stimuli that appear clearly distinct to them. Thus, no attentional effect was observed with the processing of other people's hands because their perceptual appearance might not be distinctive enough for participants and they could not prepare in advance for their presentation. This does not seem to be the case for either familiar or unfamiliar faces, since research has shown that we are experts in processing, recognizing, and retrieving face stimuli (e.g., Bruce \& Humphreys, 1994; Le Grand, Mondloch, Maurer, \& Brent, 2003). Importantly, our own hands also seem to 
appear highly distinct to us in comparison with the hands of other people.

Existing literature on body perception suggests that our own body subserves a special processing in contrast to others' bodies, regarding both motion and static appearance displays. As previously mentioned, these studies have reported that when our own body is compared with someone else's body, self-stimuli show a perceptual advantage (Daprati et al., 2007a; Frassinetti et al., 2008; Knoblich \& Flach, 2003) and recruit specific underlying neural substrates (Calvo-Merino et al., 2006; Hodzic, Muckli, et al., 2009; Myers \& Sowden, 2008). These findings seem to indicate that our body sustains a distinct internal representation in comparison with others' bodies. Along the same lines, the self-specific advantage for endogenous attention reported here may emerge from a more distinctive and robust representation of our body in contrast to someone else's body (see Tong \& Nakayama, 1999).

Previous neuroimaging results have shown that attentional expectations activate category-specific areas on the visual ventral stream prior to stimulus presentation, which have been interpreted in the context of mental imagery and perceptual representations (Esterman \& Yantis, 2010; see also Kosslyn \& Thompson, 2003). In addition, recent fMRI studies using pattern-decoding methods have revealed that attentional mechanisms can activate targetspecific neural subpopulations in the visual cortex when stimuli are not concurrently in view. The activation pattern observed in the latter studies closely resembles the neural activity triggered by stimulus-driven input; its level of specificity discriminates between different letters and grating orientations (Harrison \& Tong, 2009; Serences, Ester, Vogel, \& Awh, 2009; Stokes, Thompson, Cusack, \& Duncan, 2009; Stokes, Thompson, Nobre, \& Duncan, 2009). On this basis, it has been claimed that attentional biases are mediated via preparatory activation of relevant perceptual representations in the visual cortex with a high degree of specificity (Stokes, Thompson, Nobre, \& Duncan, 2009).

In light of these findings, it is proposed that the expectations about specific hand stimuli may imply the activation of their corresponding perceptual representations, which would help participants anticipate the target stimulus. Since the validity effect is observed exclusively for one's own hands, we suggest that the representation of our own hands is more distinct and robust than the representation of someone else's hands (see Tong \& Nakayama, 1999). In addition, the analysis of hand type (right vs. left) did not yield any significant difference in the present study. This suggests that participants did not focus on a particular feature of one of their hands (or the other person's hands) and that they used the cue to represent the personal features that define both the left and right hands as belonging to a particular identity.

In conclusion, the present results show that attention can be devoted to the specific features that define particular stimuli within a same perceptual category when those particular stimuli are remarkably distinctive for the participants. It is suggested that one's own hands sustain a distinct and more robust perceptual representation in com- parison with someone else's hands. This might have led to the emergence of an attentional effect only on self-stimuli. Nevertheless, further research on several aspects would be desirable. First, it would be relevant to test whether the observed self-specific attentional effect holds for nonbodily items when they belong or do not belong to the participants. It would also be interesting to explore how idiosyncratic attentional expectations modulate the different levels of target processing by means of electrophysiological recordings. Finally, further research would be crucial to better characterize the distinctiveness arising from the internal representation we hold about our own body and other people's bodies.

\section{AUTHOR NOTE}

Financial support for this research was provided by the Spanish Ministry of Science and Innovation (Ministerio de Ciencia e Innovación, MICINN) through Grant SEJ2007-63645 to D.S., Grant SEJ2007-63247 to P.T., and a Ramon y Cajal programme grant (30.B5.34.0201) to M.R. This study was also supported by an FPU predoctoral grant from MICINN to C.A. We are grateful to M. Longo and E. Azañon for suggestions about the reviewed literature. Correspondence relating to this article should be addressed to C. Aranda, Dpto. Psicología Experimental, Campus de Cartuja s/n, 18071 Granada, Spain (e-mail: claraac@ugr.es).

\section{REFERENCES}

Aranda, C., Tudela, P., Madrid, E., \& Ruz, M. (2010). Category expectations: A differential modulation of the $N 170$ potential for faces and words. Manuscript submitted for publication.

BAR, M. (2003). A cortical mechanism for triggering top-down facilitation in visual object recognition. Journal of Cognitive Neuroscience, 15, 600-609. doi:10.1162/089892903321662976

Baron, A., \& Journey, J. (1989). Age differences in manual versus vocal reaction times: Further evidence. Journal of Gerontology, 44, P157-P159.

Blanke, O., \& ARZY, S. (2005). The out-of-body experience: Disturbed self-processing at the temporo-parietal junction. Neuroscientist, 11, 16-24. doi:10.1177/1073858404270885

Borkenau, P., PAelecke, M., \& Yu, R. (2010). Personality and lexical decision times for evaluative words. European Journal of Personality, 24, 123-136. doi:10.1002/per.747

Bruce, V., \& Humphreys, G. W. (1994). Recognizing objects and faces. Visual Cognition, 1, 141. doi:10.1080/13506289408402299

Calvo-Merino, B., Grèzes, J., Glaser, D. E., Passingham, R. E., \& Haggard, P. (2006). Seeing or doing? Influence of visual and motor familiarity in action observation. Current Biology, 16, 2277. doi:10.1016/j.cub.2006.10.065

Correa, Á., Lupiáñez, J., Madrid, E., \& Tudela, P. (2006). Temporal attention enhances early visual processing: A review and new evidence from event-related potentials. Brain Research, 1076, 116-128. doi:10.1016/j.brainres.2005.11.074

Damasio, A. R. (1999). The feeling of what happens: Body and emotion in the making of consciousness. New York: Harcourt Brace.

Damasio, A. [R.] (2003). Feelings of emotion and the self. In J. E. LeDoux, J. Debiec, \& H. Moss (Eds.), The self: From soul to brain (Annals of the New York Academy of Sciences, Vol. 1001, pp. 253261). New York: New York Academy of Sciences.

DAPRATI, E., \& Sirigu, A. (2002). Laterality effects on motor awareness. Neuropsychologia, 40, 1379-1386.

Daprati, E., Wriessnegger, S., \& Lacquaniti, F. (2007a). Kinematic cues and recognition of self-generated actions. Experimental Brain Research, 177, 31-44. doi:10.1007/s00221-006-0646-9

Daprati, E., Wriessnegger, S., \& Lacquaniti, F. (2007b). Knowledge of one's kinematics improves perceptual discrimination. Consciousness \& Cognition, 16, 178-188. doi:10.1016/j.concog.2006.03.001

Doose, G., \& Feyereisen, P. (2001). Task specificity in age-related slowing word production versus conceptual comparison. Journals of Gerontology, 56B, P85-P87. doi:10.1093/geronb/56.2.P85 
Esterman, M., \& Yantis, S. (2010). Perceptual expectation evokes category-selective cortical activity. Cerebral Cortex, 20, 1245-1253. doi:10.1093/cercor/bhp188

Faulkner, T. F., Rhodes, G., Palermo, R., Pellicano, E., \& FerGUSON, D. (2002). Recognizing the un-real McCoy: Priming and the modularity of face recognition. Psychonomic Bulletin \& Review, 9, 327-334.

Frassinetti, F., Maini, M., Benassi, M., Avanzi, S., Cantagallo, A., \& FARNE, A. (2010). Selective impairment of self body-parts processing in right brain-damaged patients. Cortex, 46, 322-328. doi:10.1016/j.cortex.2009.03.015

Frassinetti, F., Maini, M., Romualdi, S., Galante, E., \& Avanzi, S. (2008). Is it mine? Hemispheric asymmetries in corporeal selfrecognition. Journal of Cognitive Neuroscience, 20, 1507-1516. doi:10.1162/jocn.2008.20067

Frassinetti, F., Pavani, F., Zamagni, E., Fusaroli, G., Vescovi, M., BENASSI, M., ET AL. (2009). Visual processing of moving and static self body-parts. Neuropsychologia, 47, 1988-1993. doi:10.1016/j .neuropsychologia.2009.03.012

Gallagher, S. (2000). Philosophical conceptions of the self: Implications for cognitive science. Trends in Cognitive Sciences, 4, 14-21.

Gillihan, S. J., \& FARAH, M. J. (2005). Is self special? A critical review of evidence from experimental psychology and cognitive neuroscience. Psychological Bulletin, 131, 76-97.

Griffin, I. C., Miniussi, C., \& Nobre, A. C. (2001). Orienting attention in time. Frontiers in Bioscience, 6, D660-D671.

Harrison, S. A., \& Tong, F. (2009). Decoding reveals the contents of visual working memory in early visual areas. Nature, 458, 632-635. doi:10.1038/nature07832

Hodzic, A., Kaas, A., Muckli, L., Stirn, A., \& Singer, W. (2009). Distinct cortical networks for the detection and identification of human body. NeuroImage, 45, 1264-1271. doi:10.1016/j neuroimage.2009.01.027

Hodzic, A., Muckli, L., Singer, W., \& Stirn, A. (2009). Cortical responses to self and others. Human Brain Mapping, 30, 951-962. doi:10.1002/hbm. 20558

JAMES, W. (1890). The principles of psychology. New York: Holt.

Jokisch, D., Daum, I., \& Troje, N. F. (2006). Self recognition versus recognition of others by biological motion: Viewpoint-dependent effects. Perception, 35, 911-920.

Kircher, T. T. J., Senior, C., Phillips, M. L., Benson, P. J., BullMORE, E. T., BRAMMER, M., ET AL. (2000). Towards a functional neuroanatomy of self processing: Effects of faces and words. Cognitive Brain Research, 10, 133-144. doi:10.1016/S0926-6410(00)00036-7

KNoblich, G., \& FlaCh, R. (2003). Action identity: Evidence from self-recognition, prediction, and coordination. Consciousness \& Cognition, 12, 620-632. doi:10.1016/S1053-8100(03)00070-9

Kosslyn, S. M., \& Thompson, R. (2003). When is early visual cortex activated during visual mental imagery? Psychological Bulletin, 129, 723-746. doi:10.1037/0033-2909.129.5.723

KraUZlis, R. J., \& ADLER, S. A. (2001). Effects of directional expectations on motion perception and pursuit eye movements. Visual Neuroscience, 18, 365-376. doi:10.1017/S0952523801183033

LAUWEREYNS, J. (1998). Exogenous/endogenous control of space-based/ object-based attention: Four types of visual selection? European Journal of Cognitive Psychology, 10, 41. doi:10.1080/713752264

Le Grand, R., Mondloch, C. J., Maurer, D., \& Brent, H. P. (2003). Expert face processing requires visual input to the right hemisphere during infancy. Nature Neuroscience, 6, 1108-1112. doi:10.1038/nn1121

Loula, F., Prasad, S., Harber, K., \& Shiffrar, M. (2005). Recognizing people from their movement. Journal of Experimental Psychology: Human Perception \& Performance, 31, 210-220.
Myers, A., \& Sowden, P. T. (2008). Your hand or mine? The extrastriate body area. NeuroImage, 42, 1669-1677. doi:10.1016/j .neuroimage.2008.05.045

Nebes, R. (1978). Vocal versus manual response as a determinant of age difference in simple reaction time. Journal of Gerontology, 33, 884889. doi:10.1093/geronj/33.6.884

Neisser, U. (1988). Five kinds of self-knowledge. Philosophical Psychology, 1, 35. doi:10.1080/09515088808572924

Northoff, G., Heinzel, A., De Greck, M., Bermpohl, F., DobroWOLNY, H., \& PANKSEPP, J. (2006). Self-referential processing in our brain: A meta-analysis of imaging studies on the self. NeuroImage, 31, 440-457. doi:10.1016/j.neuroimage.2005.12.002

Posner, M. (1980). Orienting of attention. Quarterly Journal of Experimental Psychology, 32, 3-25.

PURI, A. M., \& WoJcIULIK, E. (2008). Expectation both helps and hinders object perception. Vision Research, 48, 589-597. doi:10.1016/j visres.2007.11.017

Puri, A. M., Wojciulik, E., \& Ranganath, C. (2009). Category expectation modulates baseline and stimulus-evoked activity in human inferotemporal cortex. Brain Research, 1301, 89-99. doi:10.1016/j brainres.2009.08.085

Ruz, M., \& Nobre, A. C. (2008). Attention modulates initial stages of visual word processing. Journal of Cognitive Neuroscience, 20, 17271736. doi:10.1162/jocn.2008.20119

Serences, J. T., Ester, E. F., Vogel, E. K., \& Awh, E. (2009). Stimulusspecific delay activity in human primary visual cortex. Psychological Science, 20, 207-214. doi:10.1111/j.1467-9280.2009.02276.x

Stokes, M., Thompson, R., Cusack, R., \& Duncan, J. (2009). Topdown activation of shape-specific population codes in visual cortex during mental imagery. Journal of Neuroscience, 29, 1565-1572. doi:10.1523/JNEUROSCI.4657-08.2009

Stokes, M., Thompson, R., Nobre, A. C., \& Duncan, J. (2009). Shapespecific preparatory activity mediates attention to targets in human visual cortex. Proceedings of the National Academy of Sciences, 106, 19569-19574. doi:10.1073/pnas.0905306106

Sugiura, M., Kawashima, R., NaKamura, K., Okada, K., Kato, T., NaKamura, A., ET AL. (2000). Passive and active recognition of one's own face. NeuroImage, 11, 36-48. doi:10.1006/nimg.1999.0519

Sugiura, M., Sassa, Y., Jeong, H., Miura, N., Akitsuki, Y., Horie, K., ET AL. (2006). Multiple brain networks for visual self-recognition with different sensitivity for motion and body part. NeuroImage, 32, 1905-1917. doi:10.1016/j.neuroimage.2006.05.026

Sugiura, M., Watanabe, J., Maeda, Y., Matsue, Y., Fukuda, H., \& KaWASHIMA, R. (2005). Cortical mechanisms of visual self-recognition. NeuroImage, 24, 143-149. doi:10.1016/j.neuroimage.2004.07.063

Tong, F., \& NAKAYAMA, K. (1999). Robust representations for faces: Evidence from visual search. Journal of Experimental Psychology: Human Perception \& Performance, 25, 1016-1035.

ViercK, E., \& Miller, J. (2008). Electrophysiological correlates of direct selection by color. Psychophysiology, 45, 621-631. doi:10 $.1111 / \mathrm{j} .1469-8986.2008 .00650 . \mathrm{x}$

Vossel, S., Weidner, R., Thiel, C. M., \& FinK, G. R. (2009). What is "odd" in Posner's location-cueing paradigm? Neural responses to unexpected location and feature changes compared. Journal of Cognitive Neuroscience, 21, 30-41. doi:10.1162/jocn.2009.21003

Wells, F. (1924). Vocal and manual mechanisms in choice reactions. Journal of Experimental Psychology, 7, 59-66. doi:10.1037/ h0070515

(Manuscript received January 24, 2010; revision accepted for publication May 20, 2010.) 